\title{
Collection of Aerosol Particles by a Conducting Sphere in an External Electric Field-Continuum Regime Approximation
}

\author{
PAO-KUAN WANG \\ Department of Meteorology, University of Wisconsin, Madison, Wisconsin 53706 \\ Received January 4, 1982; accepted December 13, 1982
}

\begin{abstract}
The collection of charged aerosol particles by a charged conducting sphere in a uniform electric field is theoretically investigated. Continuum regime is considered and particle concentration is assumed to be described by the convective diffusion equation. Analytical solutions satisfying boundary conditions for different regions are obtained. These solutions include the simultaneous effects of Brownian motion, static charges, and external electric field. Some numerical results are presented. It is also shown that these solutions can be reduced to various limiting cases where some effects are absent.
\end{abstract}

\section{INTRODUCTION}

The collision and coagulation process of aerosol particles has long been an important problem in colloidal science. It has applications in both atmospheric sciences and engineering. In the atmosphere, for example, the collection mechanism plays an important role in the scavenging of aerosol particles. This mechanism has recently received considerable attention (1-10). In problems of chemical engineering particle coagulation is of importance as it determines to a considerable extent the size distribution and thus the reaction rate of the reactants.

In this paper we want to investigate the collection process of aerosol particles by a spherical collector in the continuum regime. The concentration of particles is assumed to be described by the convective diffusion cquation since the particles involved move by Brownian diffusion while simultaneously under the influence of convective forces such as gravity, hydrodynamic forces, and possibly phoretic and electric forces. In this study we shall consider the case where the external forces involved are electric forces.

\section{PHYSICS AND MATHEMATICS}

1. The convective diffusion of aerosol particles around an absorbing sphere in conservative force fields. In this section we will briefly describe the general formulation of the convective diffusion of small particles in the presence of conservative force fields which will be used later. Consider a cloud of monodispersed aerosol particles around a perfect absorbing sphere under the influence of a net conservative force field $\mathbf{F}$. Then by the definition of conservativeness,

or

$$
\nabla \times \mathbf{F}=0
$$

$$
\mathbf{F}=-\nabla V,
$$

i.e., it is possible, in this case, to find a scalar potential function $V$ whose gradient will give the force.

We further assume that this potential function satisfies the Laplace equation, i.e.,

$$
\nabla^{2} V=0 \text {. }
$$

Equation [3] is satisfied by many force fields such as those involved in the irrotational flow of a perfect fluid, surface waves, electromagnetic phenomena, and gravitation. 
If we assume steady-state condition, then the convective diffusion equation for particles exposed the above force field is (12)

$$
D \nabla^{2} n-B \mathbf{F} \cdot \nabla n=0,
$$

where $n$ is the concentration, $D$ the diffusivity, and $B$ the mobility of the particles, respectively. According to Eq. [2], Eq. [4] can be rewritten as:

$$
\nabla^{2} n+\frac{B}{D} \nabla V \cdot \nabla n=0
$$

The general solution of Eq. [5] is

$$
n=C_{1} \exp \left(-\frac{B V}{D}\right)+C_{2},
$$

where $C_{1}$ and $C_{2}$ are arbitrary constants to be fixed by boundary conditions. One simply has to substitute Eq. [6] into Eq. [5] to see that it is indeed a solution of Eq. [5]. By suitable transformation of Eqs. [5] and [6], one can obtain other related equations and thcir solutions (see Appendix A).

Note that the above derivations were carried out by vector operations. Thus they are valid not only for spherical coordinates but for other coordinates as well. In fact, the applications of Eqs. [5] and [6] had been made by (13) for cylindrical coordinates and by (14) for oblate spherical coordinates. Note also that the force fields which satisfy the above derivations need not to be radially symmetrical.

2. Distribution of aerosol particles around a spherical conductor under the influence of Brownian motions, electric charges, and a uniform electric field. We now consider the specific case where a conducting sphere of radius $\alpha$ is surrounded by a cloud of monodispersed acrosol particles with radius $r_{\mathrm{p}}$ (see Fig. 1). Electric charges on the sphere and the particles are $Q$ and $q$, respectively. We will assume $q>0, Q<0$ for the convenience of discussion although the solution we shall derive is valid for general cases. The Coulomb force due to the static charges is thus attractive. This force is in the radial direction $\hat{r}$ :

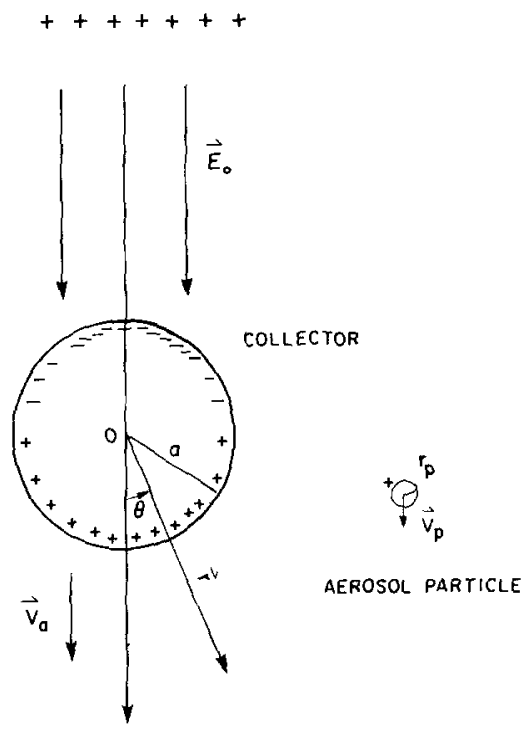

FiG. 1. Configuration of the problem.

$$
\mathbf{F}_{\mathrm{c}}=\frac{Q q}{r^{2}} \hat{r},
$$

where we have neglected the image force (see Appendix B for justification). In addition, when an external electric field $\mathbf{E}_{0}$ is present each particle will feel an additional force

$$
\mathbf{F}_{\mathrm{E}}=q \mathbf{E},
$$

where $\mathbf{E}$ is the actual electric field due to the perturbation of the sphere. In writing Eq. [8], we have assumed that (i) electrically the aerosol particles behave like point charges, and (ii) the electric field caused by the space charges $n q$ is negligible. These assumptions are justified if the particles are small compared to the collector sphere and if the charges residing on aerosol particles are small. The total external force acting on an aerosol particle is

$$
\mathbf{F}=\mathbf{F}_{\mathrm{E}}+\mathbf{F}_{\mathrm{c}}=q \mathbf{E}+\frac{Q q}{r^{2}} \hat{r},
$$

where

$$
\begin{aligned}
E=E_{0}\left(1+\frac{2 a^{3}}{r^{3}}\right) & \cos \theta \hat{r} \\
& -E_{0}\left(1-\frac{a^{3}}{r^{3}}\right) \sin \theta \hat{0} .
\end{aligned}
$$


It is easy to verify that $\nabla \times \mathbf{F}=0$ which says that the force is conservative. Thus we can write

$$
\mathbf{F}=-\nabla V .
$$

It is also simple to verify that

$$
\nabla^{2} V=0
$$

in spherical coordinates.

The concentration $n$ of the aerosol particles satisfies the steady-state convective diffusion equation

$$
D \nabla^{2} n+B \nabla V \cdot \nabla n=0
$$

which is identical with Eq. [5]. Thus the general solution is given by [6], i.e.,

$$
n=C_{1} \exp \left(-\frac{B}{D} V\right)+C_{2} .
$$

The potential $V$ is

$$
V=-\left[q E_{0}\left(r-\frac{a^{3}}{r^{2}}\right) \cos \theta-\frac{Q q}{r}\right]
$$

(see, e.g. (15)). Therefore,

$$
\begin{aligned}
n=C_{1} \exp \left\{\frac{B}{D}\right. & {\left[q E_{0}\left(r-\frac{a^{3}}{r^{2}}\right)\right.} \\
& \left.\left.\times \cos \theta-\frac{Q q}{r}\right]\right\}+C_{2} .
\end{aligned}
$$

In order to determine $C_{1}$ and $C_{2}$, we have to impose the boundary conditions. The conventional boundary conditions are (see, e.g. (16))

$$
\left\{\begin{array}{lll}
n=0 & \text { at } & r=a \\
n=n_{\infty} & \text { at } & r \rightarrow \infty
\end{array}\right.
$$

While this set of boundary conditions continues to be valid in general, it is inadequate in determining $C_{1}$ and $C_{2}$ uniquely. Some additional conditions are necessary. To find these additional boundary conditions we have to examine the distribution of the force potential, i.e., Eq. [15]. This is shown in Fig. 2. We see here that: (i) when $\cos \theta<0$, the potential increases with $r$ and the electric force is attractive everywhere; (ii) when $\cos \theta>0$, the potential increases with $r$ initially (Region-I), reaches a maximum at $r$ $=r_{\mathrm{m}}$, and then decreases with $r$ again (Region-II). Clearly, the electric force is attrac- tive in Region-I, repulsive in Region-II, and zero at $r=r_{\mathrm{m}}$. Note that $r_{\mathrm{m}}$ is not a constant but depends on $\theta$ as well as the relative magnitudes of the two competing electric fields, namely, the Coulomb field and the dipole field. The value of $r_{\mathrm{m}}$ is determined by the following equation:

$$
E_{0}\left(1+\frac{2 a^{3}}{r_{\mathrm{m}}^{3}}\right) \cos \theta=-\frac{Q}{r_{\mathrm{m}}^{2}}
$$

or

$$
r_{\mathrm{m}}^{2}+\frac{2 a^{3}}{r_{\mathrm{m}}}=-\frac{Q}{E_{0} \cos \theta},
$$

and the solution is

$$
\begin{aligned}
r_{\mathrm{m}}= & 2 \sqrt{\frac{|Q|}{3 E_{0} \cos \theta}} \\
& \times \cos \left\{\frac{1}{3} \cos ^{-1}\left[-a^{3}\left(\frac{|Q|}{3 E_{0} \cos \theta}\right)^{-3 / 2}\right]\right\} .
\end{aligned}
$$

A plot of $r_{\mathrm{m}}$ verus $\theta$ is shown in Fig. 3 for several selected values of $\lambda=|Q| /\left(E_{0} a^{2}\right.$ $\times \cos \theta$ ). It is evident from Fig. 3 that RegionI cannot cover the whole hemisphere when $\lambda<3$. This criterion can also be derived from Eq. [19] by requiring that $r \geqslant a$. In the extreme case when $E_{0} \rightarrow \infty, r_{\mathrm{m}}$ will not exist and the potential maximum will not occur outside the hemisphere.

We will now examine the solutions in two regions:

(i) When $\cos \theta<0$.

The boundary conditions are Eq. [17]:

$$
\begin{array}{lll}
n=0 & \text { at } & r=a \\
n=n_{\infty} & \text { at } & r \rightarrow \infty
\end{array}
$$

and therefore

$$
\begin{aligned}
& C_{1} \approx \frac{n_{\infty}}{\epsilon-\exp \left(-\frac{B Q q}{D a}\right)} \\
& C_{2} \approx \frac{-n_{\infty} \exp \left(-\frac{B Q q}{D a}\right)}{\epsilon-\exp \left(-\frac{B Q q}{D a}\right)},
\end{aligned}
$$


where

$$
\epsilon=\exp \left(-\frac{B q}{D} \phi_{\infty}\right)=\exp (-\infty) \rightarrow 0
$$

is a very small number, infinitely close to zero everywhere in this region except when $\cos \theta$ is itself infinitely close to zero. In the latter case $\epsilon$ is close to one. Thus $C_{1}$ and $C_{2}$ given above are not strictly constants but very nearly so. The solution is

$$
\begin{aligned}
n & \approx n_{\infty} \frac{\exp \left\{\frac{q B}{D}\left[E_{0}\left(r-\frac{a^{3}}{r^{2}}\right) \cos \theta-\frac{Q}{r}\right]\right\}-\exp \left(-\frac{B Q q}{D a}\right)}{\epsilon-\exp \left(-\frac{B Q q}{D a}\right)} \\
& \approx n_{\infty}\left(1-\exp \left\{\frac{B q}{D}\left[E_{0}\left(r-\frac{a^{3}}{r^{2}}\right) \cos \theta+\left(\frac{Q}{a}-\frac{Q}{r}\right)\right]\right\}\right) .
\end{aligned}
$$

(ii) When $\cos \theta \geqslant 0, r \leqslant r_{\mathrm{m}}$

When $\cos \theta \geqslant 0$, the conditions [17] do not determine $C_{1}$ and $C_{2}$ uniquely, and additional conditions are necessary. For this purpose, we note that the concentration gradient is given by taking the radial derivative of Eq. [16]:

$$
\begin{gathered}
\frac{\partial n}{\partial r}=C_{1} \frac{B}{D}\left[E_{0} q\left(1+\frac{2 a^{3}}{r^{3}}\right) \cos \theta+\frac{Q q}{r^{2}}\right] \\
\times \exp \left\{\frac{B}{D}\left[q E_{0}\left(r-\frac{a^{3}}{r^{2}}\right) \cos \theta-\frac{Q q}{r}\right]\right\} .
\end{gathered}
$$

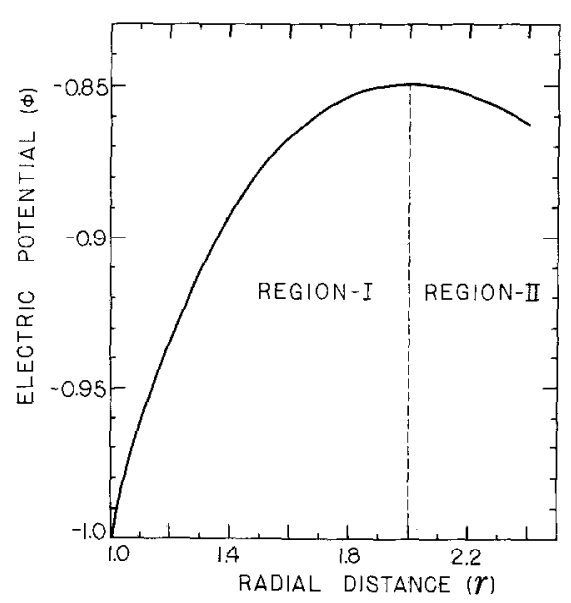

FIG. 2. The electric potential $\phi$ as a function of the radial distance $r$ for the case $a=1, Q=-1,\left|\mathbf{E}_{0}\right|=0.2$, and $\theta=0$.
At $\cos \theta=0$, the radial component of the external field is zero and we would expect that the radial concentration gradient would be the same as the case when the external field is absent. The expression of $\partial n / \partial R$ for the latter case can be found in (8). Thus,

$$
\begin{aligned}
\left.\frac{\partial n}{\partial r}\right|_{\cos \theta=0} & =C_{1} \frac{B}{D}\left[\frac{Q q}{r^{2}}\right] \exp \left(-\frac{B Q q}{D r}\right) \\
& =\frac{n_{\infty} \frac{B}{D}\left[\frac{Q q}{r^{2}}\right] \exp \left(-\frac{B Q q}{D r}\right)}{1-\exp \left(-\frac{B Q q}{D a}\right)}
\end{aligned}
$$

and therefore

$$
C_{1}=\frac{n_{\infty}}{1-\exp \left(-\frac{B Q q}{D a}\right)} .
$$

The other boundary condition is $n=0$ at $r$ $=a$, i.e.,

$$
0=C_{1} \exp \left(-\frac{B Q q}{D a}\right)+C_{2}
$$

and therefore

$$
C_{2}=-\frac{n_{\infty} \exp \left(-\frac{B Q q}{D a}\right)}{1-\exp \left(-\frac{B Q q}{D a}\right)}
$$

The complete solution is therefore 


$$
n=n_{\infty} \frac{\exp \left\{\frac{B}{D}\left[E_{0} q\left(r-\frac{a^{3}}{r^{2}}\right) \cos \theta-\frac{Q q}{r}\right]\right\}-\exp \left(-\frac{B Q q}{D a}\right)}{1-\exp \left(-\frac{B Q q}{D a}\right)}
$$

At $r=r_{\mathrm{m}}$ the radial components of the external field for all $\theta$ vanish, and we would expect that $\partial n / \partial r=0$ at $r=r_{\mathrm{m}}$ for all $\theta$. But this requirement is automatically satisfied in view of Eq. [26] since the quantity in the first square brackets vanishes at $r=r_{\mathrm{m}}$.

Equation [30] can be written as

$$
n=n_{\infty} \frac{\exp \left(-\frac{B Q q}{D a}\right)-\exp \left\{\frac{B}{D}\left[E_{0} q\left(r-\frac{a^{3}}{r^{2}}\right) \cos \theta-\frac{Q q}{r}\right]\right\}}{\exp \left(-\frac{B Q q}{D a}\right)-1}
$$

Both the numerator and denominator on the right hand side of Eq. [31] are now positive. It is easy to see that the concentration is not uniform at $r=r_{\mathrm{m}}$ (remember that $r_{\mathrm{m}}$ is a function of $\theta$ ). The concentration $n_{r=r_{m}}$ is largest at $\cos \theta=0$ since the potential is zero at $r=r_{\mathrm{m}}$. On the other hand, the smallest $n_{r=r_{\mathrm{m}}}$ occurs at $\theta=0$ since the potential maximum is lowest and therefore the negative of the potential maximum is largcst (see Fig. 2).

Even at a constant $r$ near the sphere, the

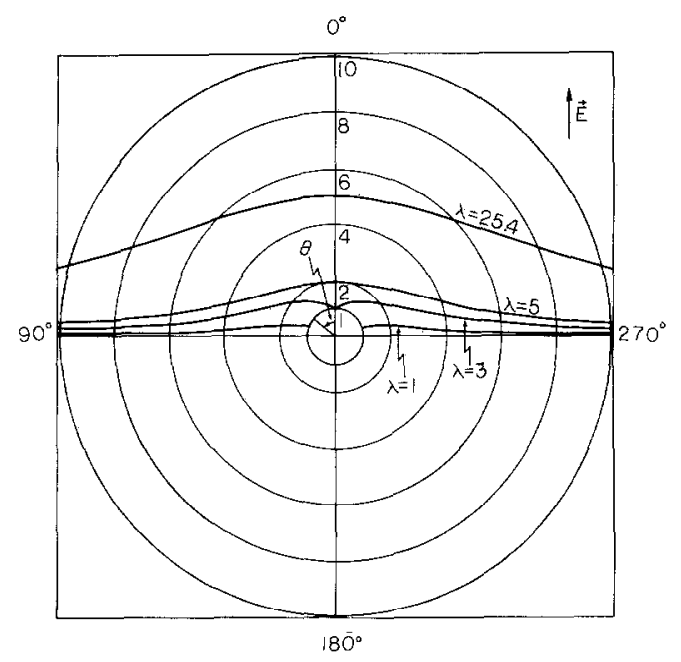

FIG. 3. The surfaces of $r=r_{\mathrm{m}}$ calculated from Eq. [20] for different values of $\lambda$. Note that these surfaces are not equipotential surfaces. concentration $n$ is also smallest along $\theta=0$, as can be seen from Eq. [31]. This is consistent with the physical consideration because this is where the particles meet the strongest repulsive forces.

From Eq. [31] we can also see that when $E_{0}$ increases to an extent that the second term in the numerator equals the first term, the concentration becomes zero. Further increase in $E_{0}$ would cause the concentration to become negative. The negative value itself is unimportant; it simply means that under very strong external field, particles cannot come close to the hemisphere because of the very strong repulsive forces. This is also consistent with physical reasonings.

The solution for the region where $\cos \theta$ $\geqslant 0$ and $r>r_{\mathrm{m}}$ is not obtained yet. On the other hand it should not concern us here because the particle flux in this region is basically drifting along the field lines and is directed away from the sphere. Thus, insofar as the collection of aerosol particles by the sphere is concerned, we can ignore this region.

3. The calculation of collection kernel. To calculate the collection kernel, we need to know the radial concentration gradients at the surface of the sphere. Thus by taking the radial derivatives of Eqs. [25] and [31] and set $r=a$, we obtain. 


$$
\left.\frac{\partial n}{\partial r}\right|_{\substack{\cos \theta<0 \\ r=a}}=-n_{\infty} \frac{B}{D}\left[3 E_{0} q \cos \theta+\frac{Q q}{a^{2}}\right]
$$

and

$$
\begin{aligned}
\left.\frac{\partial n}{\partial r}\right|_{\substack{\cos \theta \geqslant 0 \\
r=a}}=-n_{\infty} \frac{\frac{B}{D}\left[3 E_{0} q \cos \theta+\frac{Q q}{a^{2}}\right] \exp \left(-\frac{B Q q}{D a}\right)}{\exp \left(-\frac{B Q q}{D a}\right)-1} \\
=-n_{\infty} \frac{\frac{B}{D}\left[3 E_{0} q \cos \theta+\frac{Q q}{a^{2}}\right]}{1-\exp \left(\frac{B Q q}{D a}\right)} .
\end{aligned}
$$

The collection kernel is

$$
\begin{aligned}
K=-\frac{1}{n_{\infty}} \frac{\partial N}{\partial t}=\oint_{r=\mathbf{a}} D \nabla n \cdot d \mathbf{A}=K_{1}+K_{2} \\
=\int_{0}^{2 \pi} \int_{0}^{\pi / 2} \frac{B\left[3 E_{0} q \cos \theta+\frac{Q q}{a^{2}}\right]}{1-\exp \left(\frac{B Q q}{D a}\right)} a^{2} \sin \theta d \theta d \phi+\int_{0}^{2 \pi} \int_{\pi / 2}^{\pi} B\left[3 E_{0} q \cos \theta+\frac{Q q}{a^{2}}\right] a^{2} \sin \theta d \theta d \phi \\
=\frac{-\left(3 \pi E_{0} a^{2} B q+2 \pi B Q q\right)}{1-\exp \left(\frac{B Q q}{D a}\right)}+\left(3 \pi E_{0} a^{2} B q-2 \pi B Q q\right) .
\end{aligned}
$$

The two terms in Eq. [34] should be evaluated separately before adding together. Negative values of $K_{1}$ or $K_{2}$ should be regarded as zero since it simply means that no particle is collected.

The above derivation is based on the assumption that the surface $r=r_{\mathrm{m}}$ extends outside the sphere. In the case when $\lambda<3$, this surface does not cover the whole hemisphere but covers only from $\theta=\theta_{\mathrm{c}}$ to $\theta=\pi / 2$ (see Fig. 3). In this case, the lower limit of the inner integral in $K_{1}$ is not 0 but should be replaced by $\theta_{\mathrm{c}}$. The resulted collection kernel is

where

$$
K=\frac{-\left[\frac{3}{2} \pi E_{0} a^{2} B q\left(1+\cos 2 \theta_{c}\right)+2 \pi B Q q \cos \theta_{c}\right]}{1-\exp \left(\frac{B Q q}{D a}\right)}+\left(3 \pi E_{0} a^{2} B q-2 \pi B Q q\right),
$$

$$
\theta_{\mathrm{c}}=\cos ^{-1}\left(\frac{|Q|}{3 E_{\mathrm{u}} a^{2}}\right) \text {. }
$$

The above formulation is valid for stationary collectors. For collections moving in a viscous medium the hydrodynamic forces should be considered. The calculations which include hydrodynamic forces are, however, quite involved. Examples of this type of calculations were given in (6) where the Brownian motion was not considered. Alternatively an approximated method which takes the hydrodynamic effects into account was given by 
(10). There a ventilation factor was used to represent the overall enhancement of the particle flux. Thus according to this approximation the collection kernel is given by

$$
K=\frac{-\left(3 \pi E_{0} a^{2} B q+2 \pi B Q q\right)}{1-\exp \left(\frac{B Q q}{\bar{f}_{\mathrm{p}} D a}\right)}+\left(3 \pi E_{0} a^{2} B q-2 \pi B Q q\right)
$$

for $\lambda \geqslant 3$ and

$$
K=\frac{-\left[\frac{3}{2} \pi E_{0} a^{2} B q\left(1+\cos 2 \theta_{\mathrm{c}}\right)+2 \pi B Q q \cos \theta_{\mathrm{c}}\right]}{1-\exp \left(\frac{B Q q}{\tilde{f}_{\mathrm{p}} D a}\right)}+\left(3 \pi E_{0} u^{2} B q-2 \pi B Q q\right)
$$

for $\lambda<3$. The previous paper (10) must be consulted for the formulas of various ventilation factors.

\section{RESULTS AND DISCUSSIONS}

Equations [34]-[38] were used to calculate the collection kernels of aerosol particles of radii 0.001 to $1.0 \mu \mathrm{m}$ by spheres of 10 to 400 $\mu \mathrm{m}$ in the presence of external electric fields of strength 100 to $3000 \mathrm{~V} / \mathrm{cm}$. All computations were carried out for $900 \mathrm{mbar}$ and $10^{\circ} \mathrm{C}$. The spheres and particles were assumed to carry electric charges of

$$
\begin{aligned}
Q & =2 a^{2} \mathrm{esu} \\
q & =2 r_{\mathrm{p}}^{2} \mathrm{esu},
\end{aligned}
$$

respectively, with $a$ and $r_{\mathrm{p}}$ in centimeters. This size dependence of electric charges is that proposed in (17) for the mean thunderstorm charges. Note that for particles of radii smaller than about $0.15 \mu \mathrm{m}$ the above formulas give values of charges smaller than one electron $\left(\sim 4.8 \times 10^{-10} \mathrm{esu}\right)$. The values thus represent only the average charges of a cloud of mixed charged and neutral particles. Since the charges of small particles are usually very small, we assume here that, for the case when $q$ is smaller than one electron, the aerosol cloud is composed of particles carrying either one electron charge or no charge. The fraction of charged particles is therefore given by

$$
R_{\mathrm{q}}=\frac{2 r_{\mathrm{p}}^{2}}{4.8 \times 10^{-10}}
$$

whereas the fraction of neutral particles is given by

$$
R_{\mathrm{B}}=1-R_{\mathrm{q}} \text {. }
$$

The neutral particles are assumed to be unaffected by the electric forces and thus perform pure Brownian motions with the collection kernel

$$
K_{\mathrm{B}}=4 \pi D a .
$$

The total kernel is therefore

$$
K=R_{\mathrm{q}} K_{\mathrm{q}}+\left(1-R_{\mathrm{q}}\right) K_{\mathrm{B}},
$$

where $K_{\mathrm{q}}$ is the collection kernel of charged particles given by one of the Eqs. [34]-[38].

Figures 4 to 8 show the collection kernels of aerosol particles by stationary spheres of various sizes in the presence of electric fields of $100,500,1000,2000$, and $3000 \mathrm{~V} / \mathrm{cm}$, respectively. Figures 9 to 13 show the collection kernels of the same set of particle and sphere sizes, except that now the ventilation effect is considered. The two sets of figures look similar to each other except in the magnitudes. The general feature is that the kernel decreases with aerosol size initially, reaches a minimum in the size range between 0.01 and $0.1 \mu \mathrm{m}$, and then increases with particle size. This general feature can be understood in terms of the relative importance of the Brownian motion and the electric effect. For very small particles the Brownian motion is 


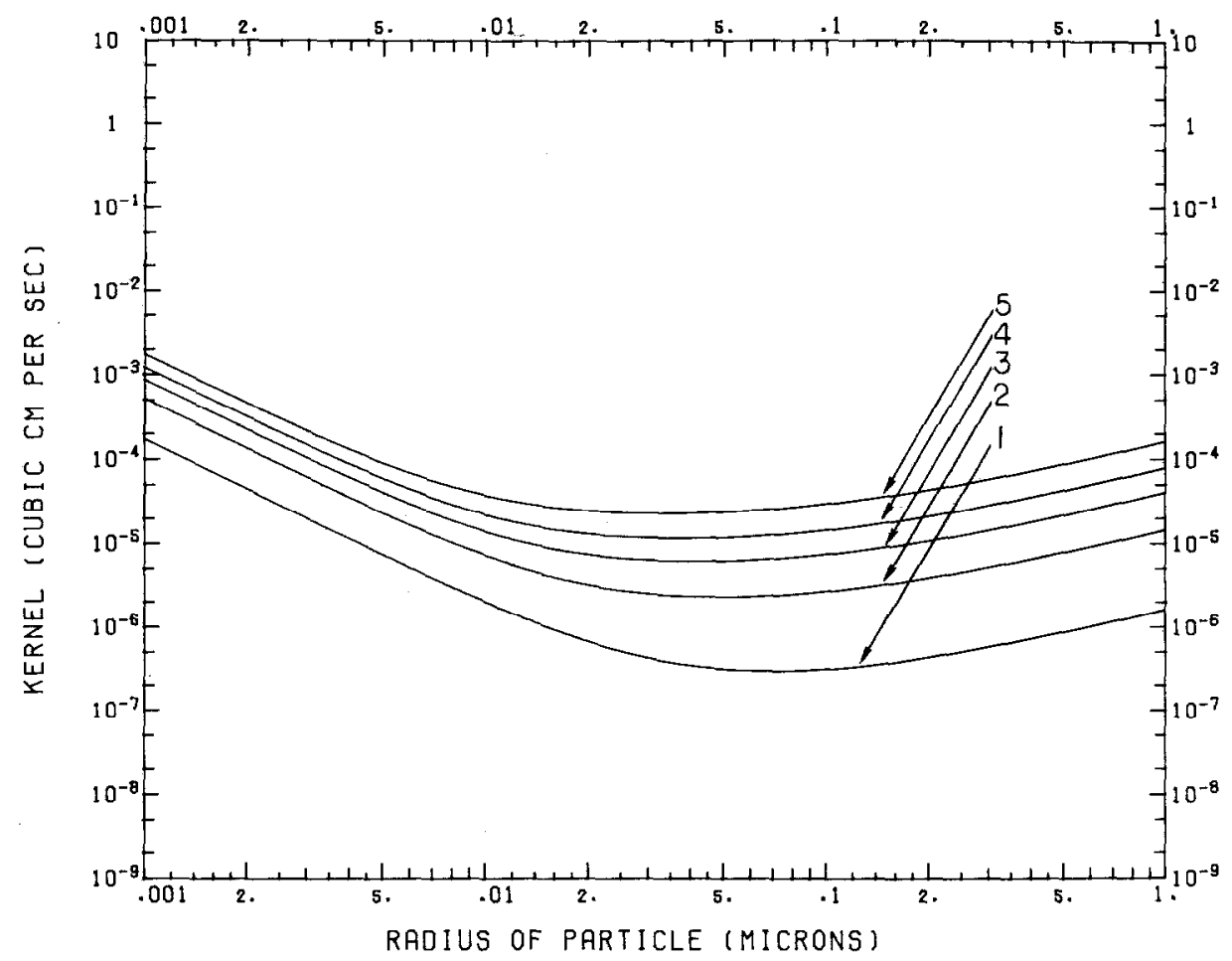

FIG. 4. Collection kernels of aerosol particles captured by spheres of various sizes due to Brownian diffusion and electric forces in air of $900 \mathrm{mbar}$ and $10^{\circ} \mathrm{C}$ and external electric field $E_{0}=100 \mathrm{~V} / \mathrm{cm}$. (1) $a=10 \mu \mathrm{m}$, (2) $a=30 \mu \mathrm{m}$, (3) $a=50 \mu \mathrm{m}$, (4) $a=70 \mu \mathrm{m}$, (5) $a=100 \mu \mathrm{m}$. No ventilation effect. $Q=2 a^{2}$ esu, $q=2 r_{p}^{2}$ esu, $a$ and $r_{\mathrm{p}}$ in centimeters.

dominant, the effect being larger for smaller particles, thus the kernel decreases when size increases. On the other hand, the electric effect is dominant for large particle sizes, the effect being larger for larger particles due to the larger charges. Thus the kernel increases with size at this end. In the middle neither the Brownian motion nor the electric effect is strong, the kernel is therefore a minimum here. A more detailed discussion is given in (10).

Figures 14 and 15 compare the collection kernels of aerosol particles captured by spheres of radii 30 and $100 \mu \mathrm{m}$ for the cases with and without ventilation effect. Clearly the ventilation effect is stronger for smaller aerosol particles while the larger particles $(r>0.1 \mu \mathrm{m})$ are hardly affected. This can be realized in terms of the larger inertia of the particles. In addition the ventilation effect is stronger for the larger collector $(a=100 \mu \mathrm{m})$ because of larger hydrodynamic forces.

Figure 16 compares the kernels obtained by the present formulation and that of (10) for collectors of radii 30 and $106 \mu \mathrm{m}$ in the electric field $\mathbf{E}_{0}=3000 \mathrm{~V} / \mathrm{cm}$. As can be seen here the differences between the two are not very large, being about $10-20 \%$ in most cases. The present formulation gives a lower value than that in (10). This is to be expected since the formulation in (10) simply adds the flux due to Brownian diffusion to that due to electric effect, thus implicitly assuming that the Brownian diffusion is not affected by the electric field. This is of course less accurate. The present formulation, on the other hand, considers the reduction of the Brownian flux in the presence of the electric field and is therefore more physically realistic. The small difference between the two, however, does 


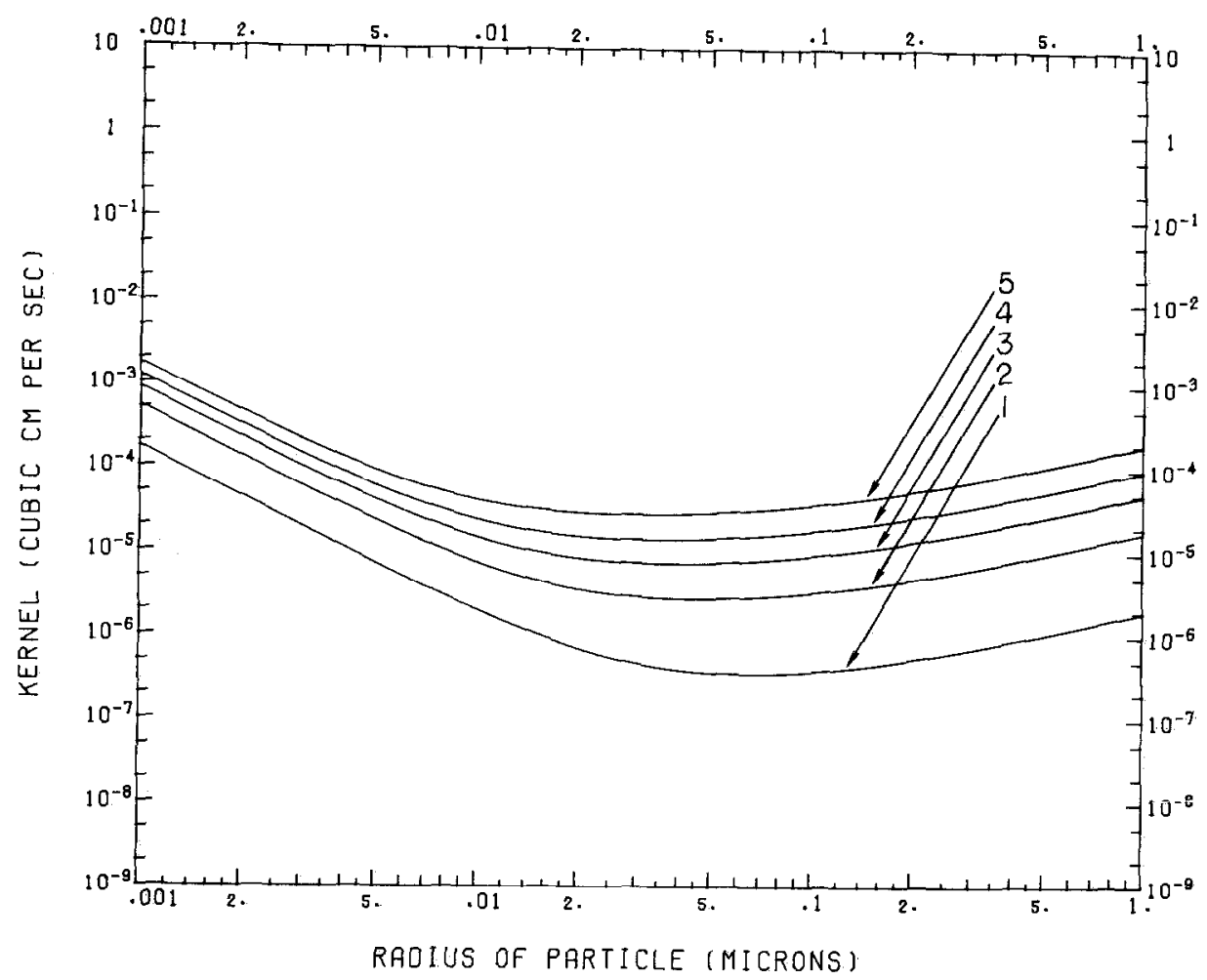

FIG. 5. Same as Fig. 4 except for $E_{0}=500 \mathrm{~V} / \mathrm{cm}$.

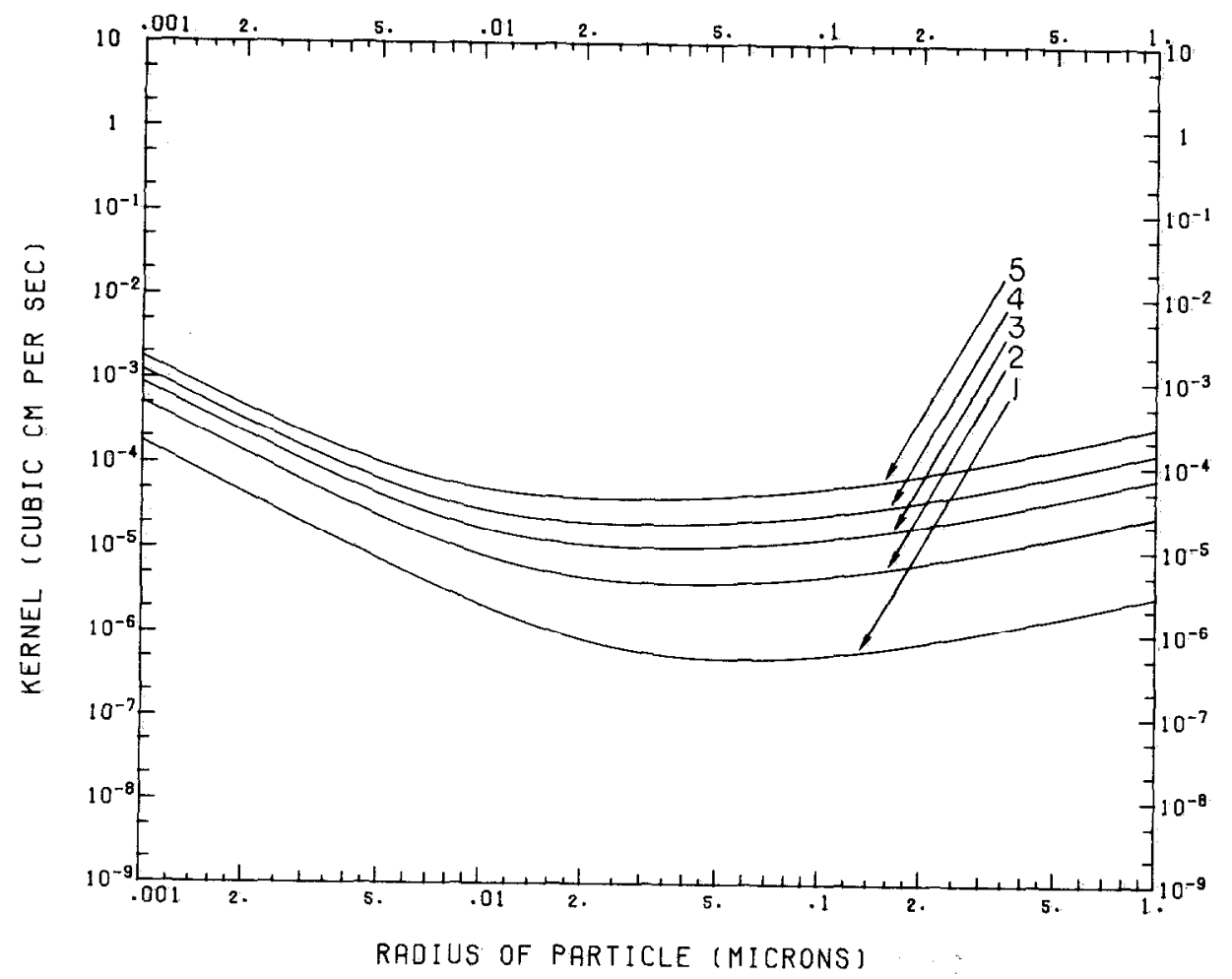

FIG. 6. Same as Fig. 4 except for $E_{0}=1000 \mathrm{~V} / \mathrm{cm}$. 


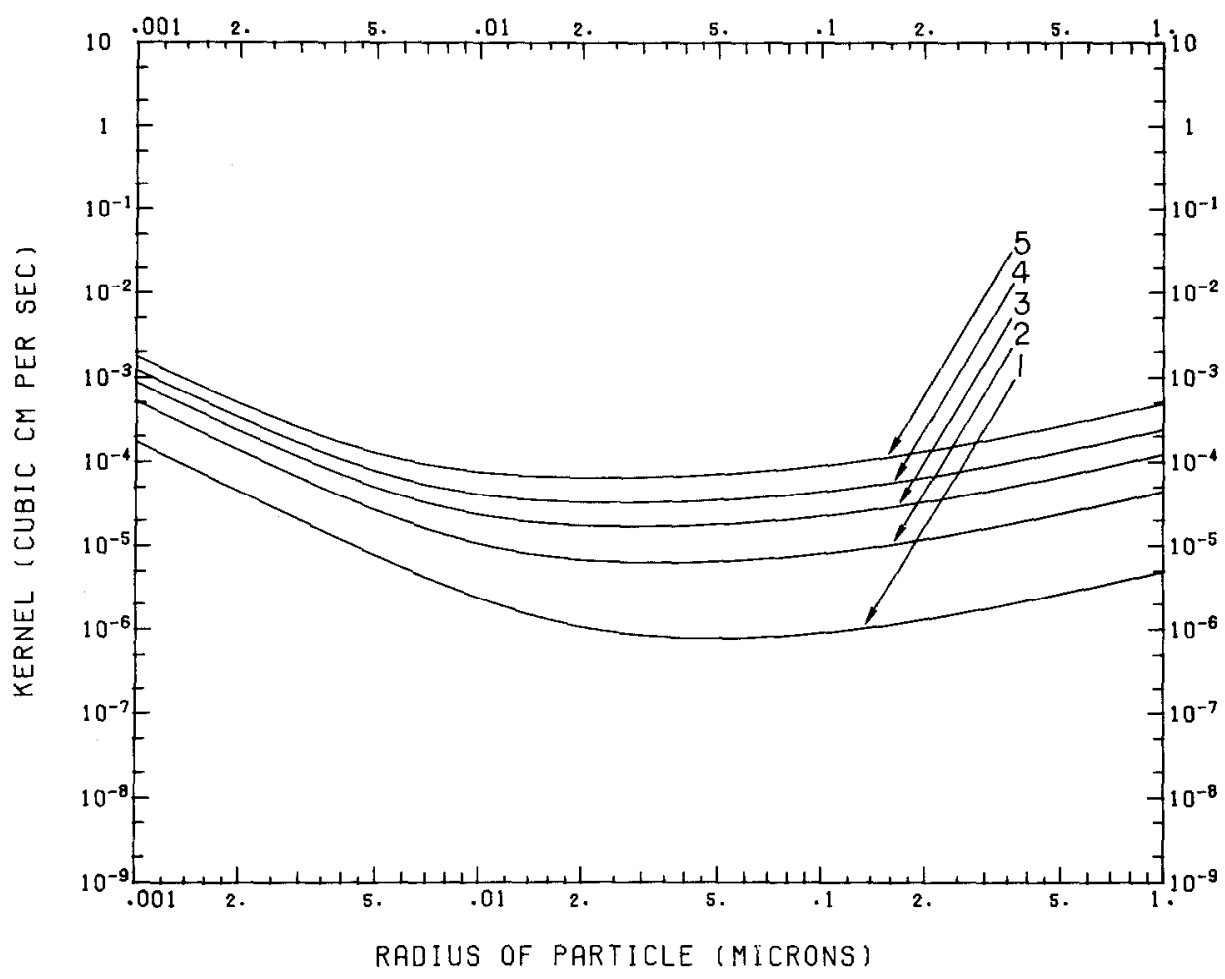

FIG. 7. Same as Fig. 4 except for $E_{0}=2000 \mathrm{~V} / \mathrm{cm}$.

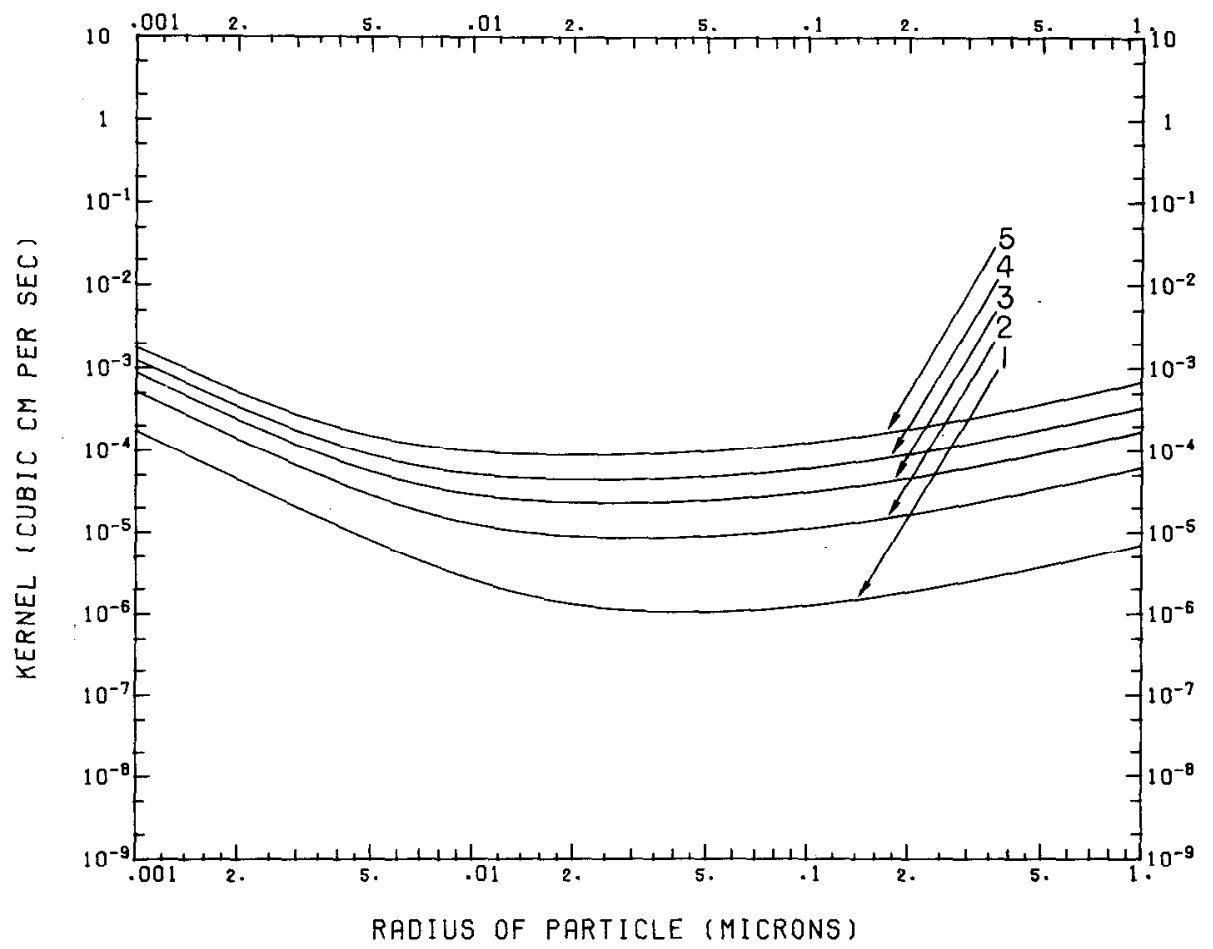

Fig. 8. Same as Fig. 4 except for $E_{0}=3000 \mathrm{~V} / \mathrm{cm}$. 


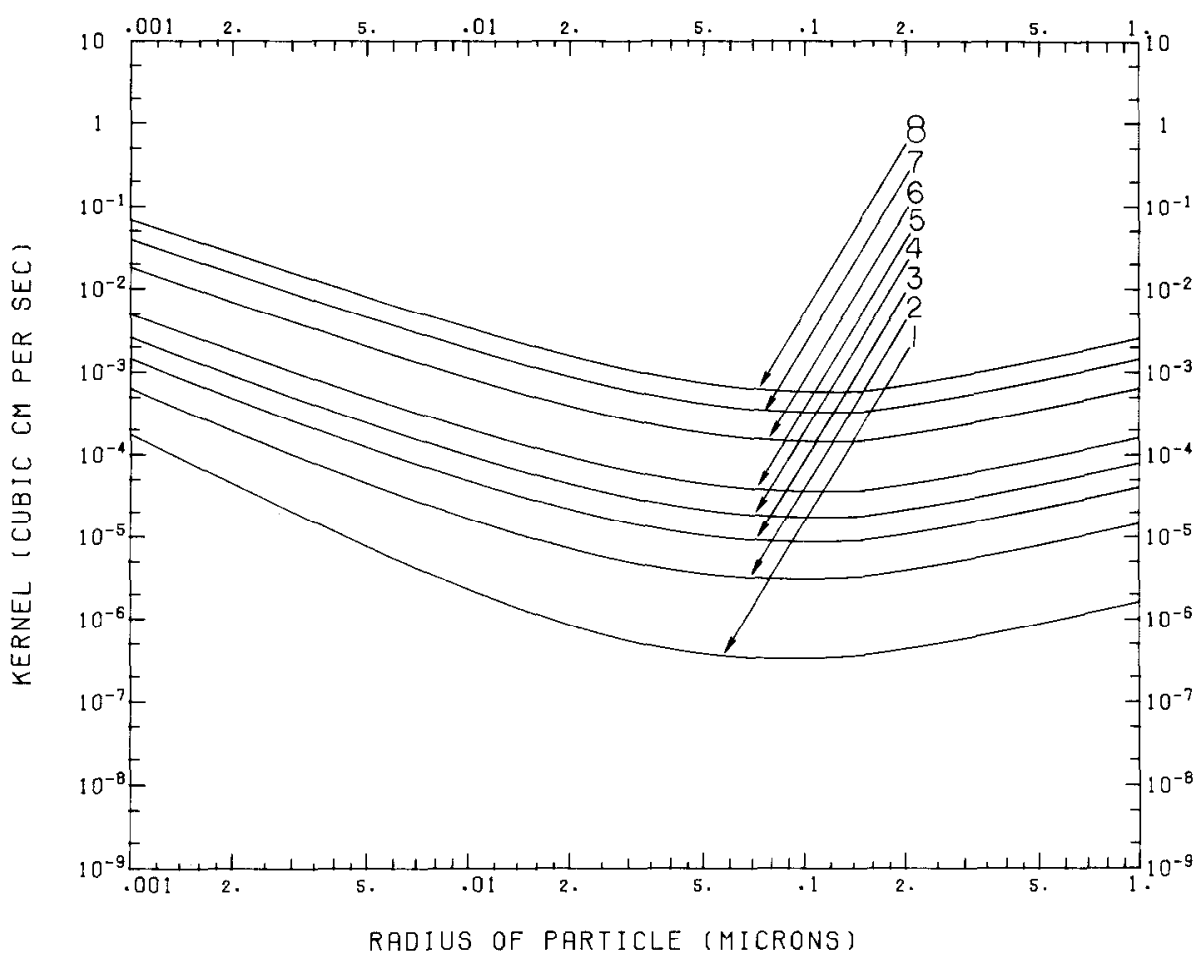

FIG. 9. Collection kernels of aerosol particles captured by spheres of various sizes due to Brownian diffusion and electric forces in air of 900 mbar and $10^{\circ} \mathrm{C}$ and external electric field $E_{0}=100 \mathrm{~V} / \mathrm{cm}$. (1) $a=10 \mu \mathrm{m}$, (2) $a=30 \mu \mathrm{m}$, (3) $a=50 \mu \mathrm{m}$, (4) $a=70 \mu \mathrm{m}$, (5) $a=100 \mu \mathrm{m}$, (6) $a=200 \mu \mathrm{m}$, (7) $a=300 \mu \mathrm{m}$, (8) $a=400 \mu \mathrm{m}$. Ventilation effects are included. $Q=2 a^{2}$ esu, $q=2 r_{\mathrm{p}}^{2}$ esu, $a$ and $r_{\mathrm{p}}$ in centimeters.

testify an old empirical rule that the sum of the pure Brownian flux and pure conduction current represents a good approximation for the total flux in many cases.

\section{a. Limiting Cases}

We shall now show that in various limiting cases the solutions in Sections 2 and 3 can be reduced and can represent the proper solutions for these limits.

(i) When there is no external field ( $E_{0}$ $=0$ ). In this case, $\epsilon=1$ in Eq. [23] instead of zero, and Eq. [24] becomes exactly

$$
n=n_{\infty} \frac{\exp \left(-\frac{B Q q}{D r}\right)-\exp \left(-\frac{B Q q}{D a}\right)}{1-\exp \left(-\frac{B Q q}{D a}\right)}
$$

while Eq. [30] also reduces exactly to this result. This is identical with the solution obtained by (8) where the particle distribution is determined only by the Brownian diffusion and central forces such as that caused by static charges.

(ii) Pure Brownian diffusion. When both electric forces due to the external field and the electric charges are absent, then the particle distribution is solely determined by the Brownian diffusion. We can obtain this from the above solutions.

Since there is no external electric field, the starting equation will be the same as Eq. [44]. We then take the limit of Eq. [44] when $Q$ and $q$ are infinitely approaching zero. Thus, by expanding the exponential functions in [44] into power series we obtain 


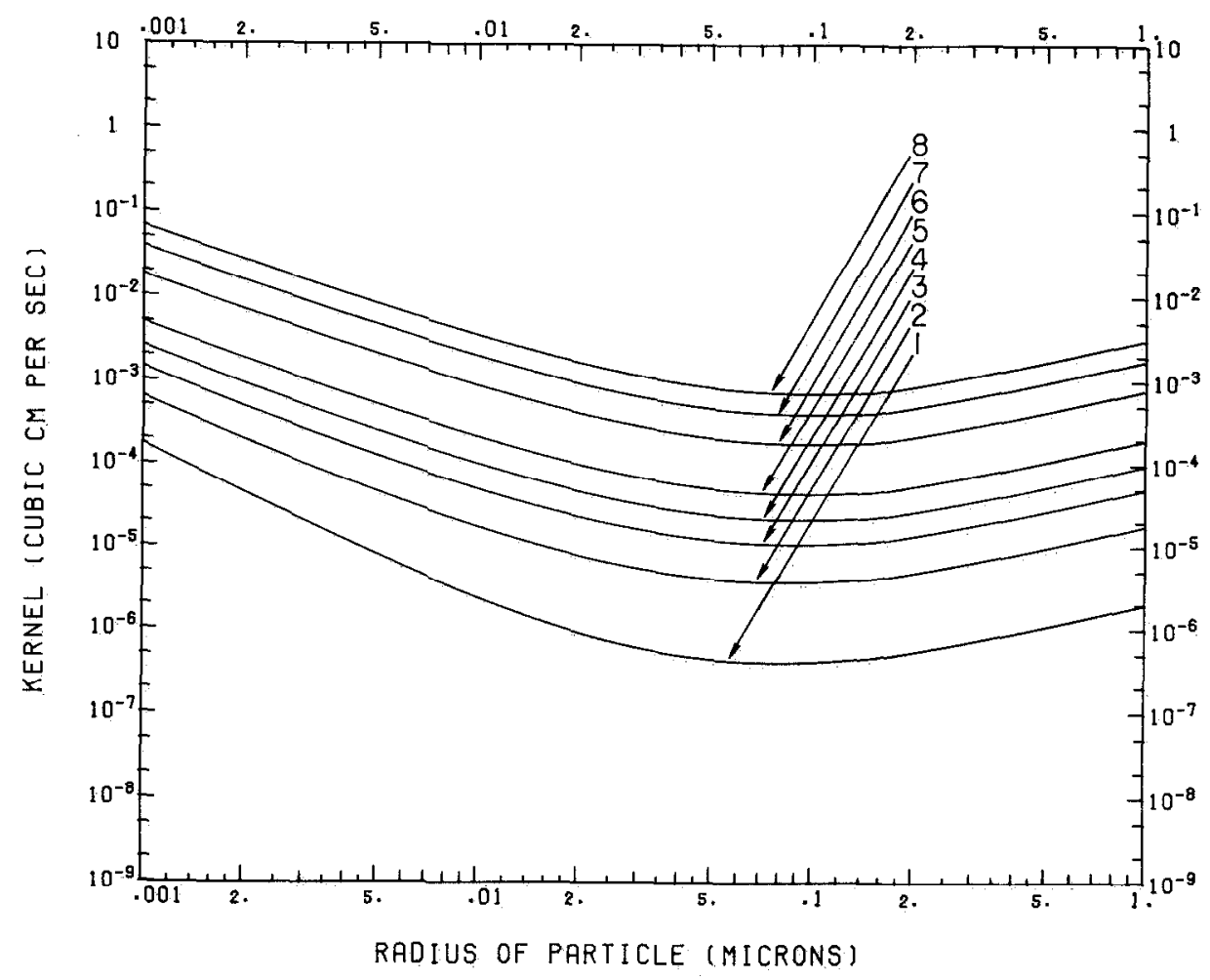

FIG. 10. Same as Fig. 9 except for $E_{0}=500 \mathrm{~V} / \mathrm{cm}$.

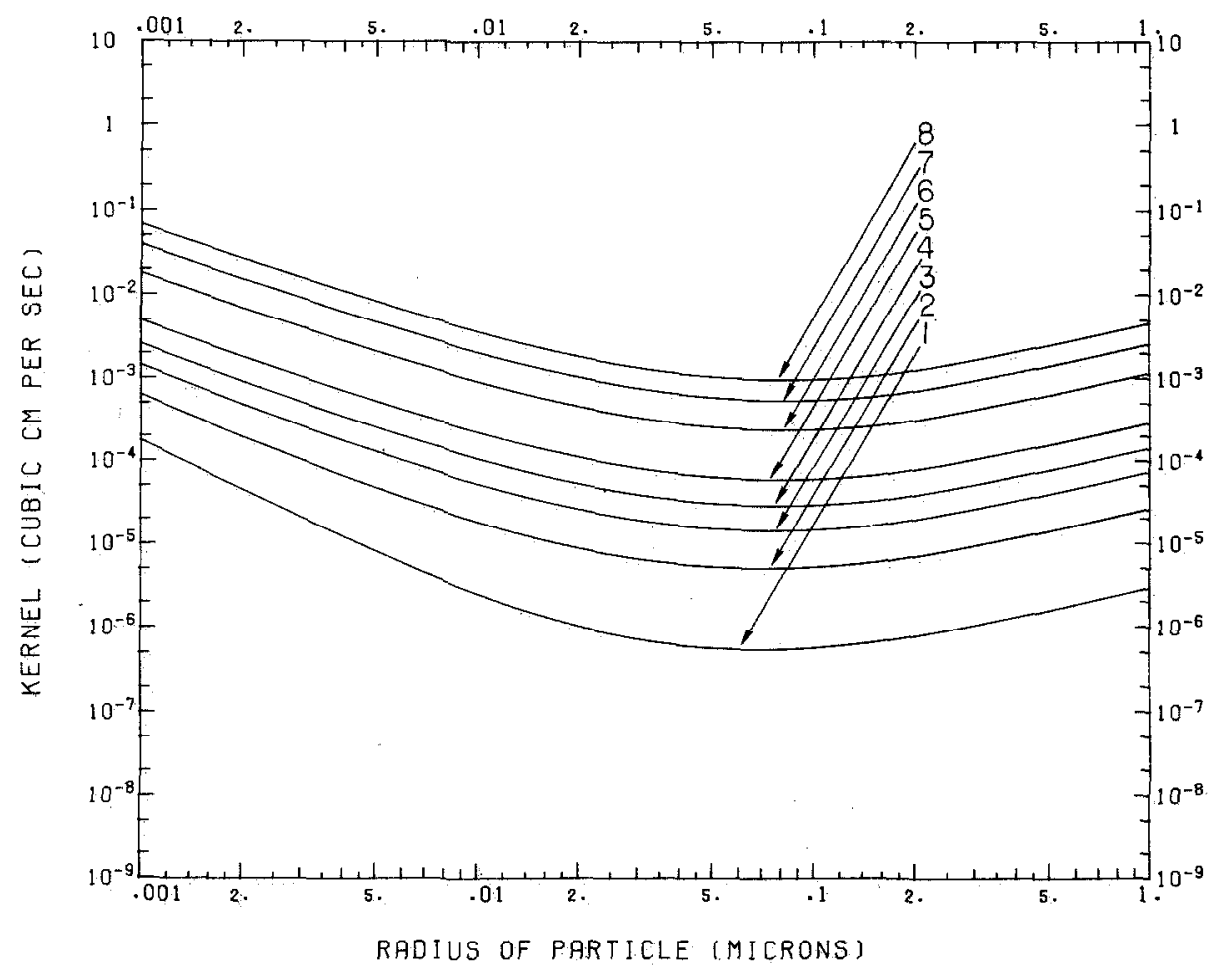

Fig. 11. Same as Fig. 9 except for $E_{0}=1000 \mathrm{~V} / \mathrm{cm}$. 


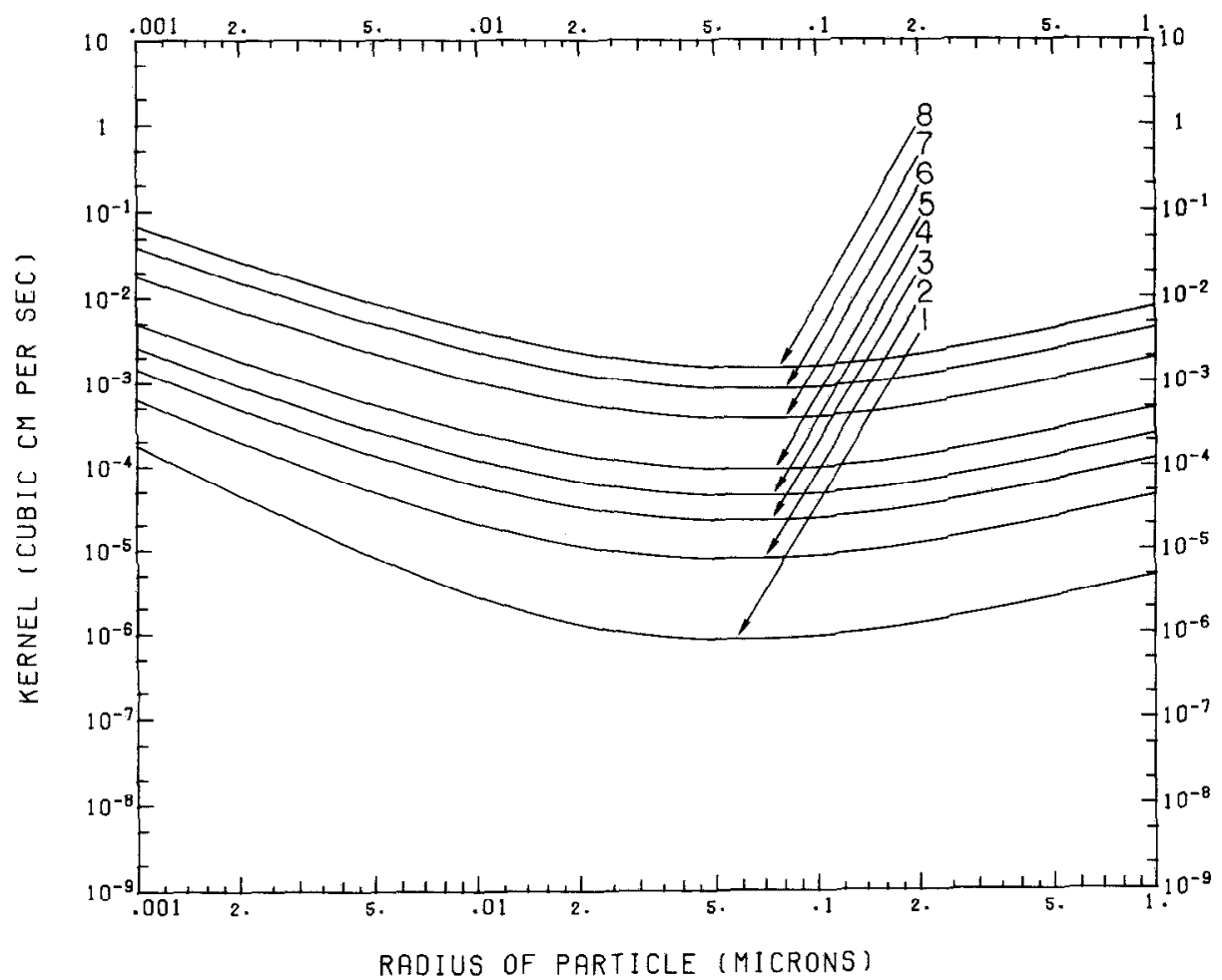

FIG. 12. Same as Fig. 9 except for $E_{0}=2000 \mathrm{~V} / \mathrm{cm}$.

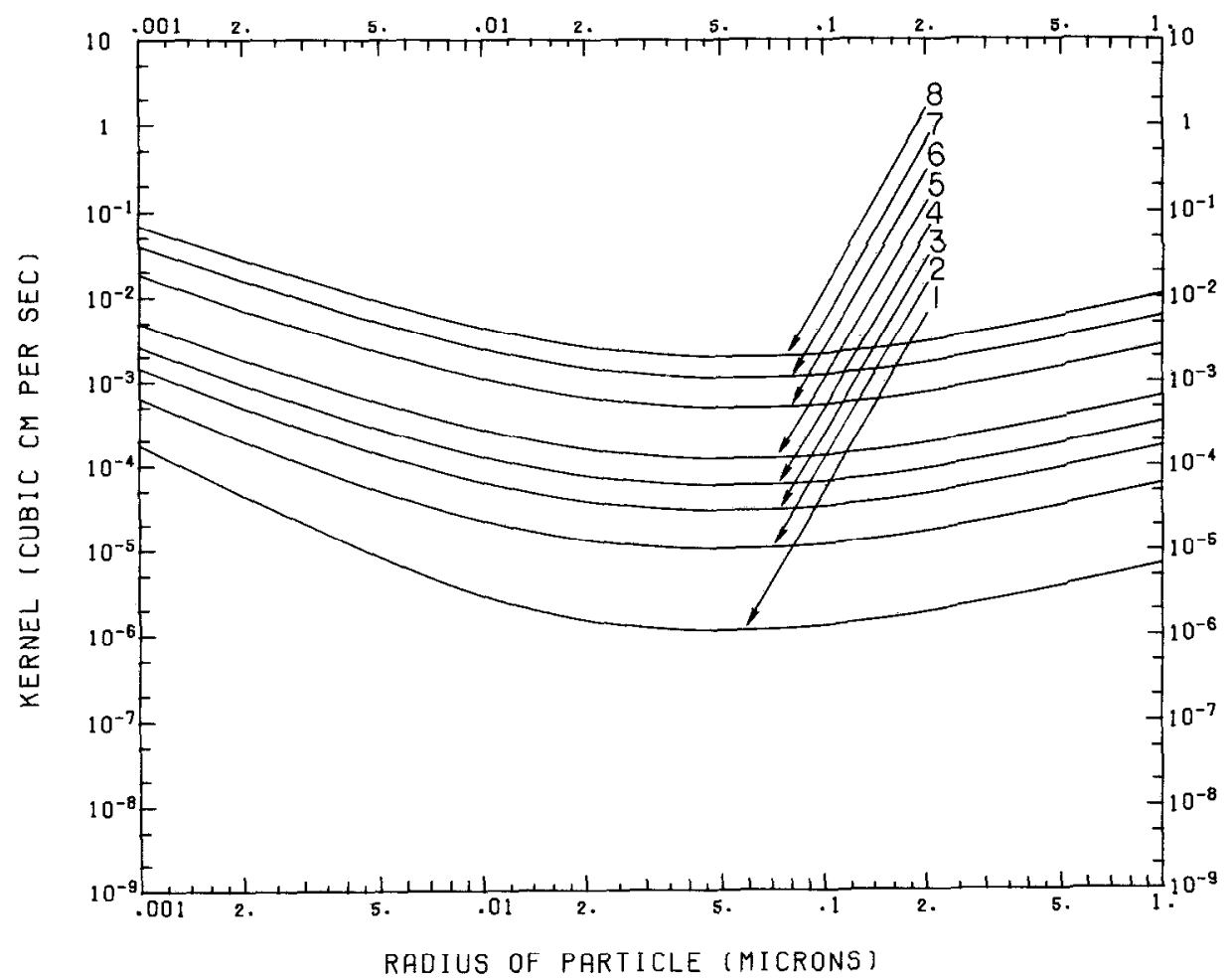

FiG. 13. Same as Fig. 9 except for $E_{0}=3000 \mathrm{~V} / \mathrm{cm}$. 


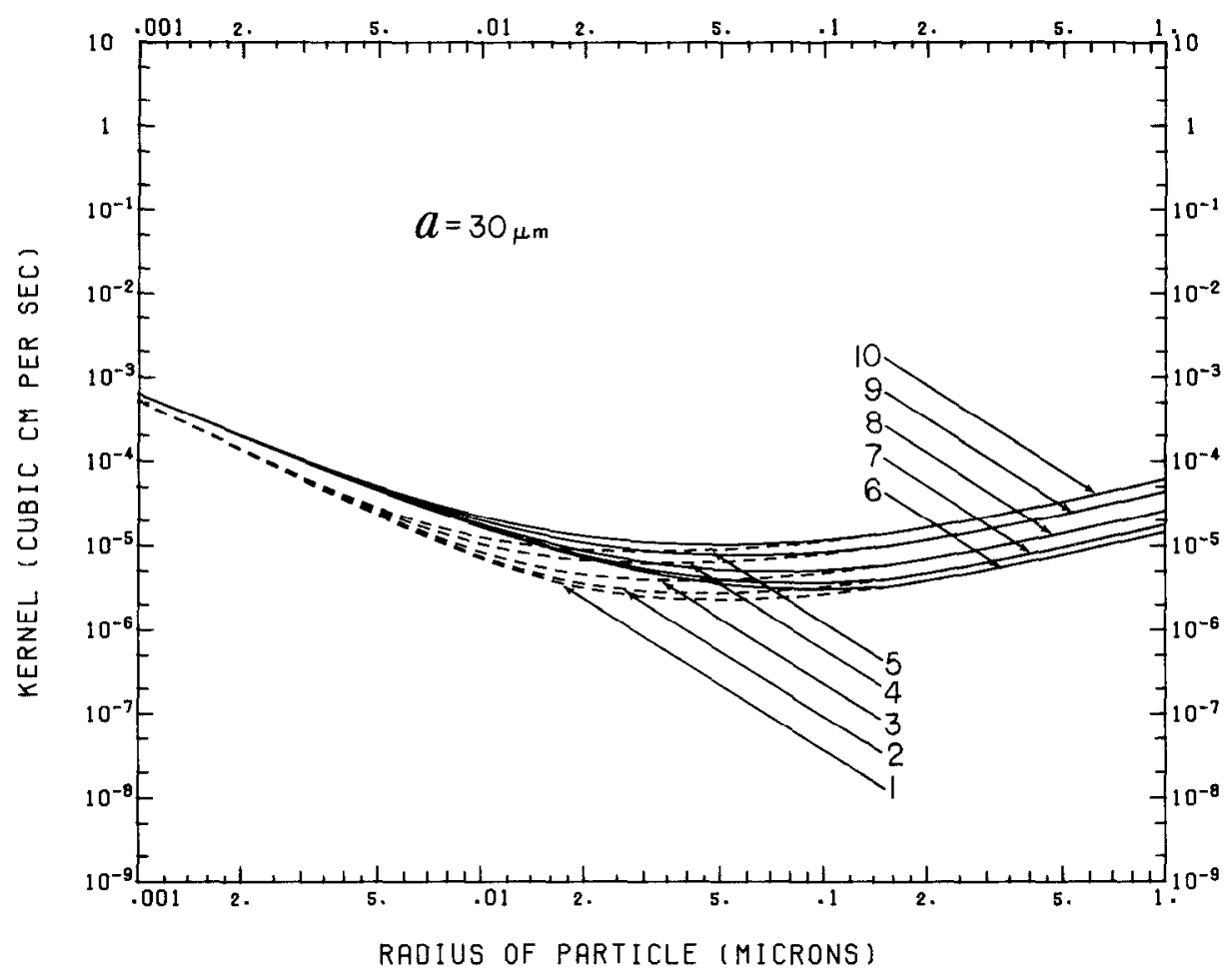

FIG. 14. Comparison between the collection kernels of aerosol particles captured by spheres of radius $a=30 \mu \mathrm{m}$ with (-) and without (-- ) ventilation effects. Curves (1)-(5) are for cases without ventilation effects and $E_{0}=100,500,1000,2000$, and $3000 \mathrm{~V} / \mathrm{cm}$, respectively. Curves (6)-(10) are for cases with ventilation effects and $E_{0}=100,500,1000,2000$, and $3000 \mathrm{~V} / \mathrm{cm}$.

$$
\begin{aligned}
\underset{\substack{Q \rightarrow 0 \\
q \rightarrow 0}}{n}=\lim _{\substack{Q \rightarrow 0 \\
q \rightarrow 0}} n_{\infty}\left[\frac{\exp \left(-\frac{B Q q}{D r}\right)-\exp \left(-\frac{B Q q}{D a}\right)}{1-\exp \left(-\frac{B Q q}{D a}\right)}\right] \\
=\lim _{\substack{Q \rightarrow 0 \\
q \rightarrow 0}} n_{\infty}\left[\frac{\left.\frac{B Q q}{D a}\left(1-\frac{a}{r}\right)+\frac{1}{2 !}\left(\frac{B Q q}{D a}\right)^{2}\left(1-\frac{a^{2}}{r^{2}}\right)+\cdots\right]}{\frac{B Q q}{D a}-\frac{1}{2 !}\left(\frac{B Q q}{D a}\right)^{2}+\cdots}\right] \\
=\lim _{\substack{Q \rightarrow 0 \\
q \rightarrow 0}} n_{\infty}\left[\frac{\frac{B Q q}{D a}\left(1-\frac{a}{r}\right)}{\frac{B Q q}{D a}}\right] \text { (neglect higher order terms) } \\
=\lim _{\substack{Q \rightarrow 0 \\
q \rightarrow 0}} n_{\infty}\left(1-\frac{a}{r}\right)=n_{\infty}\left(1-\frac{a}{r}\right) .
\end{aligned}
$$




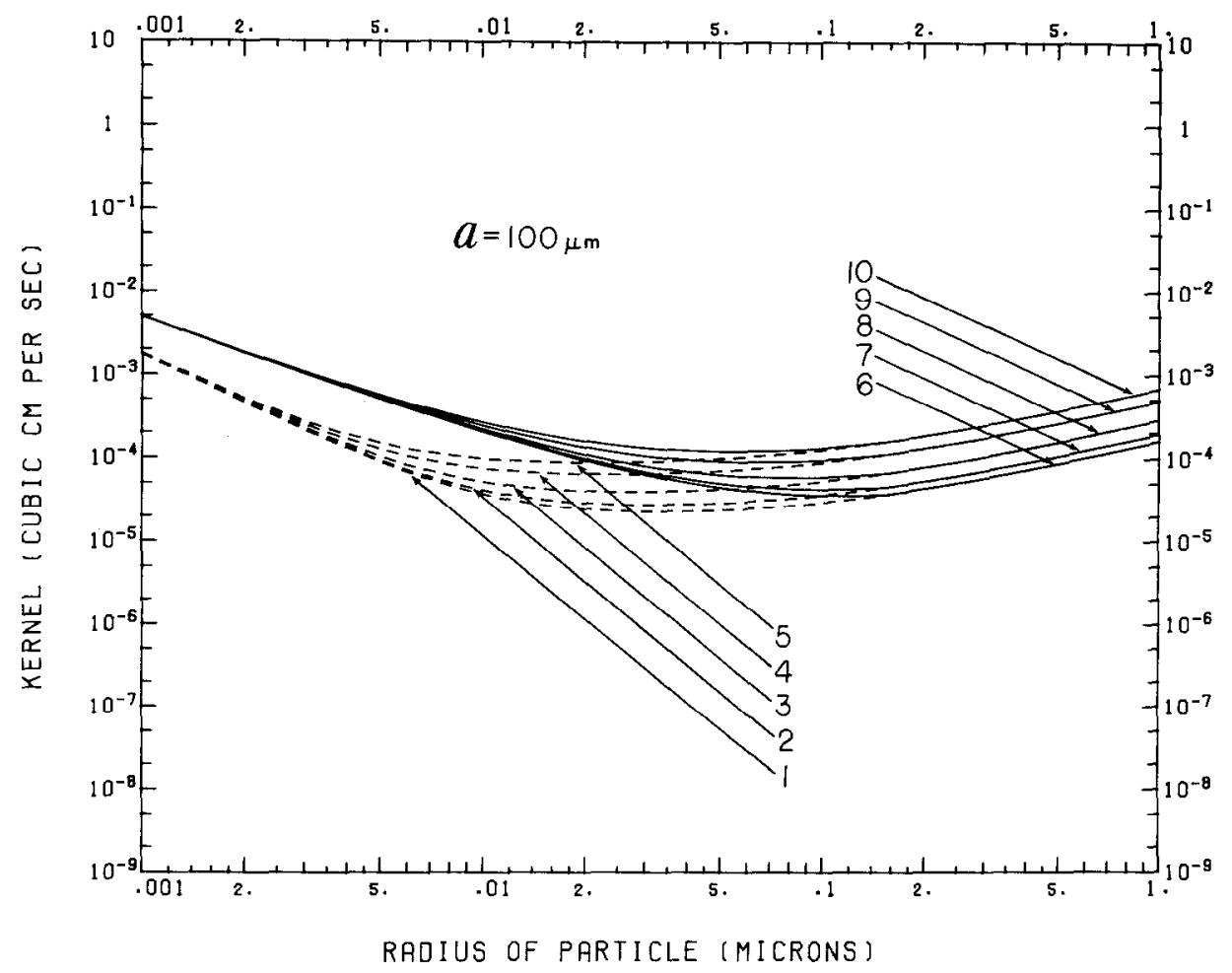

Fig. 15. Same as Fig. 14 except for $a=100 \mu \mathrm{m}$.

Equation [45] is the well-known particle distribution around a sphere due to pure Brownian motions (see, e.g. (8)).

(iii) Very strong external electric field. When the external field is so strong as to predominate the whole process, the particle distribution is solely determined by the external field. The total mass flux toward the sphere is the convective current due to the external field. This can be easily obtained by taking $E_{0} a^{2} \Rightarrow|Q|$ in Eq. [35]. Since $\theta_{\mathrm{c}}$ $\sim \pi / 2$ in this case, we have

$$
K \approx 3 \pi E_{0} a^{2} B q
$$

since the first term is a negative number and should be set to zero. Equation [46] is the well-known convective current due to a uniform external field $E_{0}$ (see, e.g. (18), for the case of ion transport toward a spherical drop due to $E_{0}$ ).

\section{CONCLUSIONS}

In the above discussions we have shown that the distribution of aerosol particles by a stationary, conducting sphere in the presence of an external electric field can be described by the convective diffusion Eq. [13] with solution Eqs. [25] and [31]. The particle flux is given by Eqs. [34]-[38]. We have also proved that in the limiting cases (i) $E_{0}=0$, (ii) pure Brownian diffusion, and (iii) very strong $E_{0}$, the above solutions can be reduced to proper solutions for these cases.

Although the present paper deals exclusively with the electrostatic forces, the formulation is quite general and is valid for any conservative force fields whose potentials satisfy the Laplace equation. Thus one can easily add forces such as thermo- and diffusiophoretic forces.

We want to stress, however, that we have not included the hydrodynamic forces which 


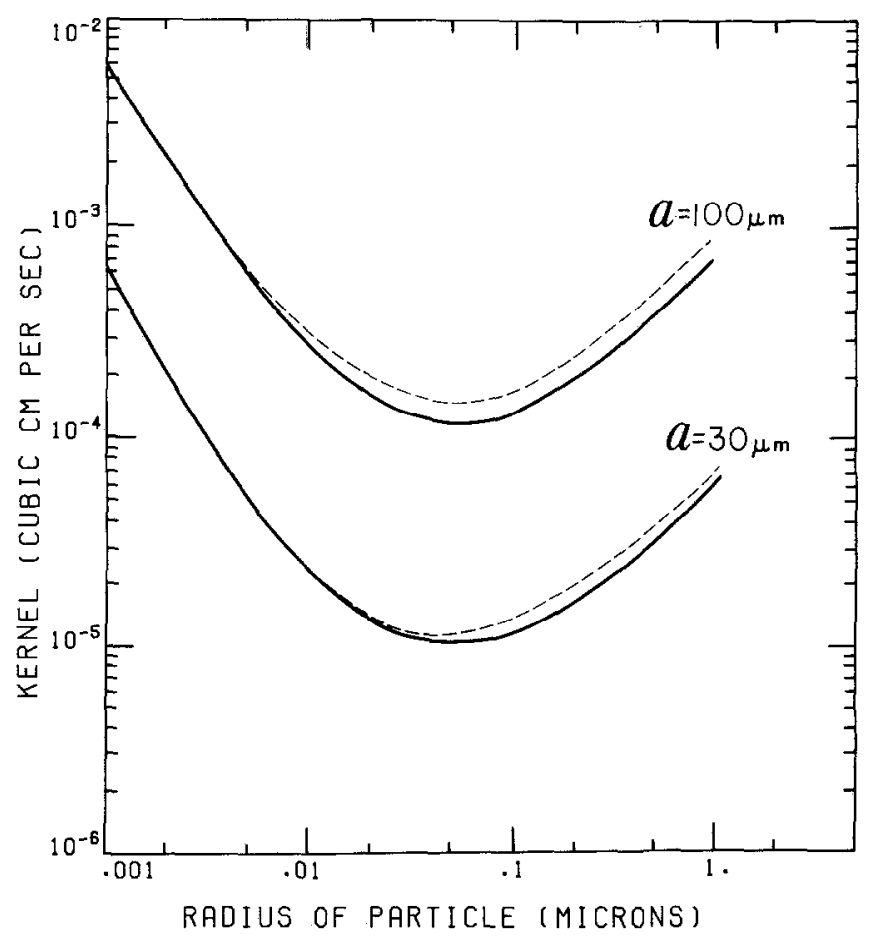

FIG. 16. Comparison between the collection kernels calculated from the present formulation and from Ref. (10). Solid curves are the present results. Dashed curves are results from Ref. (10). $E_{0}=3000 \mathrm{~V} / \mathrm{cm}$ for all cases. Only Brownian diffusion and electric forces are considered, but with ventilation effect.

are more complicated. Thus the results obtained above should not be compared directly with the experimental results which were obtained in the presence of hydrodynamic forces. We are currently working toward this direction. The present paper, in the mean time, should represent a step forward in solving the convective diffusion problem in a nonsymmetric force field.

\section{APPENDIX A}

In the following we will provide some convenient formulas for obtaining particular solutions of convective diffusion processes under the influence of conservative force fields. To our knowledge these formula have not been brought to the attention of many investigators.

Consider a vector field $\mathbf{F}$ which is conservative and satisfies Eqs. [1]-[3]. Let $\psi$ be any continuous function which is at least twice differentiable. Then

(i) the equation

$$
\nabla^{2} \psi+\mathbf{F} \cdot \nabla \psi=0
$$

has a solution

$$
\psi=C_{1} e^{v}+C_{2},
$$

(ii) the equation

$$
\nabla^{2} \psi-\mathbf{F} \cdot \nabla \psi=0
$$

has a solution

$$
\psi=C_{1} e^{-v}+C_{2},
$$

(iii) the equation

$$
\nabla^{2} \psi+\mathbf{F}^{2} \psi=0
$$

has the solution

$$
\psi=C_{1} e^{i n}+C_{2} e^{-i v} \quad\left(i^{2}=-1\right),
$$


and (iv) the equation

$$
\nabla^{2} \psi-\mathbf{F}^{2} \psi=0
$$

has a solution

$$
\psi=C_{1} e^{v}+C_{2} e^{-v},
$$

wherc $\mathbf{F}^{2}=\mathbf{F} \cdot \mathbf{F}=\nabla v \cdot \nabla v, v$ is the potential of F, defined in Eq. [2]. These can be verified by simply substituting the solutions into the corresponding equations. Equations [47] and [49] are generally regarded as the diffusion equations while Eqs. [51] and [53] are the Helmholtz equations.

Equation [53] is also mathematically identical with the time-independent Schrödinger equation.

It is interesting to note that Eqs. [47], [49], and [53] are mutually transformable. If we let $\psi=\psi^{\prime} \exp (v / 2)$ and substitute into Eq. [47] we will obtain

$$
\nabla^{2} \psi^{\prime}-\frac{1}{4}(\nabla v \cdot \nabla v) \psi^{\prime}=0
$$

or

$$
\nabla^{2} \psi^{\prime}-\left(\nabla v^{\prime} \cdot \nabla v^{\prime}\right) \psi^{\prime}=0 \quad\left(v^{\prime}=\frac{v}{2}\right)
$$

or

$$
\nabla^{2} \psi^{\prime}-\mathbf{F}^{2} \psi^{\prime}=0 \quad\left(\mathbf{F}^{\prime}=-\nabla v^{\prime}\right)
$$

which is identical with Eq. [53]. On the other hand if we substitute $\psi=\psi^{\prime} \exp (-v / 2)$ into Eq. [49], we will again obtain Eq. [55]. This type of transformation has been introduced by Fürth (1931) (cited in Eq. [12] for onedimensional case). In the above we see that one can perform the transformation in a more general three-dimensional form if the vector F satisfies Eqs. [1]-[3].

When using the above formula one has to be sure that $\mathbf{F}$ does indeed satisfy Eqs. [1][3] and that the form of the equation is indeed the same as those listed in Eqs. [47], [49], [51], and [53]. When these requirements are met, the remaining work is to find the potential function $v$ for $\mathbf{F}$. This last step can be found in many standard textbooks of mathematical physics.

\section{APPENDIX B}

The complete electric force between a point charge and a charged conducting sphere is (see, e.g. (15) or (19))

$$
|\mathbf{F}|=\frac{\mathbf{q}}{r^{2}}\left[Q-\frac{q a^{3}\left(2 r^{2}-a^{2}\right)}{r\left(r^{2}-a^{2}\right)^{2}}\right],
$$

where the second term in the brackets represents the image force. Since the aerosol particles are not really point charges but of finite sizes, they will be captured by the collector at a distance $r=a+r_{\mathrm{p}}$. At this distance the electric force is

$$
\begin{aligned}
|\mathbf{F}|=q\{ & \frac{Q}{\left(a+r_{\mathrm{p}}\right)^{2}} \\
& \left.-\frac{q a^{3}\left[a\left(a+r_{\mathrm{p}}\right)^{2}-a^{2}\right]}{\left(a+r_{\mathrm{p}}\right)\left[\left(a+r_{\mathrm{p}}\right)^{2}-a^{2}\right]^{2}}\right\} .
\end{aligned}
$$

Since the image force is larger for larger $q$ which, in turn, requires a larger particle radius in our calculation $\left(q \propto r_{\mathrm{p}}^{2}\right)$, we take the largest particle $\left(r_{\mathrm{p}}=1 \mu \mathrm{m}\right)$ and the smallest sphere ( $a=10 \mu \mathrm{m}$, so that $Q$ is smallest) for an estimate of the relative importance of the two terms. Putting these values of $a$ and $r_{\mathrm{p}}$ into Eq. [57] and assuming that $Q=2 a^{2}, q$ $=2 r_{\mathrm{p}}^{2}\left(a, r_{\mathrm{p}}\right.$ in centimeters $)$, we obtain

$$
\begin{gathered}
\frac{Q}{\left(a+r_{\mathrm{p}}\right)^{2}}=\frac{2 \times 10^{-6}}{\left(1.1 \times 10^{-3}\right)^{2}}=1.65 \\
\frac{q a^{3}\left[2\left(a+r_{\mathrm{p}}\right)^{2}-a^{2}\right]}{\left(a+r_{\mathrm{p}}\right)\left[\left(a+r_{\mathrm{p}}\right)^{2}-a^{2}\right]^{2}} \\
=\frac{2 \times 10^{-8} \times 10^{-9} \times 1.42 \times 10^{-6}}{1.1 \times 10^{-3} \times\left(0.21 \times 10^{-6}\right)^{2}} \\
=5.85 \times 10^{-7}
\end{gathered}
$$

Clearly the image force is negligibly small in comparison with the first term for aerosol particles captured by larger spheres. Thus in our calculation we can safely neglect the image force. 


\section{ACKNOWLEDGMENT}

The author thanks the anonymous reviewers for very useful suggestions and Ms. Eva Singer for typing the manuscript. This research is partially supported by the United States Environmental Protection Agency under assistance agreement R809371-01-0. Although the research described in this article has been funded in part by EPA, it has not been subject to the agency's required peer and administrative review and, therefore, does not reflect the view of the agency and no official endorsement should be inferred.

\section{RFFFRENCES}

1. Hampl, V., Kerker, M., Cooke, D. D., and Matijevič, E., J. Atmos. Sci. 28, 1221 (1971).

2. Slinn, W. G., and Hales, J. M., J. Atmos. Sci. 28, 1465 (1971).

3. Hampl, V., and Kerker, M., J. Colloid Interface Sci. 40, 305 (1972).

4. Kerker, M., and Hampl, V., J. Atmos. Sci. 31, 1368 (1974).

5. Beard, K. V., J. Atmos. Sci. 31, 1595 (1974).

6. Grover, S. N., Pruppacher, H. R., and Hamielec, A. E., J. Atmos. Sci. 34, 1655 (1977).
7. Wang, P. K., and Pruppacher, H. R., J. Atmos. Sci. 34, 1664 (1977).

8. Wang, P. K., Grover, S. N., and Pruppacher, H. R., J. Atmos. Sci. 35, 1973 (1978).

9. Lai, K. Y., Dayan, N., and Kerker, M., J. Atmos. Sci. 35, 674 (1978).

10. Wang, P. K., and Pruppacher, H. R., J. Colloid Interface Sci. 75, 286 (1980).

11. Fuchs, N. A., "The Mechanics of Aerosols." Pergamon, Elmsford, N.Y., 1964.

12. Jost, W., in "Diffusion in Solids, Liquids, and Gases." Academic Press, New York, 1960.

13. Wang, P. K., and Pruppacher, H. R., Pure Appl. Geophys. 118, 1090-1108 (1980).

14. Martin, J. J., Wang, P. K., and Pruppacher, H. R., J. Atmos. Sci. 37, 1628-1638 (1980).

15. Jackson, J. D., in "Classical Electrodynamics," 641 pp. Wiley, New York, 1962.

16. Chandrasekhar, S., Rev. Mod. Phys. 15, 1-89 (1943).

17. Takahasi, T., Rev. Geophys. Space Phys. 11, 903 (1973).

18. Whipple, F. J. W., and Chalmers, J. A., Quart. J. R. Meteor. Soc. 70, 103-120 (1944).

19. Marlow, W. H. (Ed.), "Aerosol Microphysics I: Particle Interactions," Vol. 16, 160 pp. SpringerVerlag, Berlin/Heidelberg/New York, 1980. 